\title{
Alteraciones del esquema rimático en cantigas de mestría ${ }^{1}$
}

Mercedes Brea Universidad de Santiago de Compostela

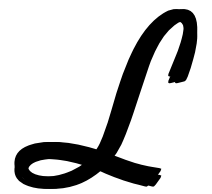

os trovadores medievales suelen repetir la estructura métrico-rimática-melódica de la primera estrofa en todas las demás que configuran una cantiga (con excepción, naturalmente, de la fiinda, que puede tener incluso una melodía diferente). Existen, sin embargo, casos en los que cada cobla es diferente (lo que caracteriza precisamente la modalidad conocida como descort), y pueden encontrarse también ejemplos de otro tipo con posibles alteraciones más o menos conscientes del esquema, además de las que puedan deberse a problemas achacables a la transmisión manuscrita. Así, en la lírica gallego-portuguesa, una de las modificaciones más frecuentes del esquema rimático se produce en las cantigas de refrán que siguen el modelo de

${ }^{1}$ Este trabajo forma parte de una investigación más amplia, realizada en colaboración con Gerardo Pérez Barcala, y se inserta en los proyectos de investigación El debate metaliterario en la lírica románica medieval (FFI2011-26785), subvencionado por el MICINN, y $O$ rei don Denis e os trobadores da súa época, financiado por la Xunta de Galicia dentro de su programa de proyectos sectoriales (09SEC023204PR). 
las cobras singulares, ${ }^{2}$ cuando en (alg)una de las estrofas una de las rimas empleadas coincide con una del refrán (sin que ello afecte, obviamente, a la unidad melódica ni a la distribución básica de las rimas). ${ }^{3}$

Los casos que vamos a revisar corresponden todos a cantigas de mestría; en ellas, las variantes que puedan encontrarse deberán ser necesariamente explicadas de modo diferente a las presentes en cantigas de refrán, y sin descartar en ningún caso la posible existencia de una transmisión defectuosa (de la que sólo daremos cuenta si es apreciable en los manuscritos conservados).

1. Osoir' Anes, en Cuidei eu de meu coraçon (111,1), combina de modo alternado las rimas -on y -ar en las dos primeras estrofas (I: $\mathrm{a}=-$ on, $\mathrm{b}=$-ar / II: $\mathrm{a}=-\mathrm{ar}, \mathrm{b}=-\mathrm{on}$ ), pero sólo en los dos primeros versos, repite la disposición de II en III y complica el esquema con la iteración de la rima $c$ (-or) en I y III (queda, así, "aislado" c = -eus en II); además, reserva siempre la rima en -ar (que funciona como $b$ en I, pero como $a$ en II y III) para el último verso de cada estrofa (convirtiendo con ello las estrofas en capcaudadas):

${ }^{2}$ Puede tratarse también de cantigas que combinan una rima singular con outra unisonante, o que repiten una de las rimas de una estrofa en posición diferente en otra. Tanto los textos que citamos como los códigos que los identifican proceden de la base de datos $M e d D B$ (www.cirp.es), que reproduce (con pequeñas adaptaciones) el RM de Tavani (TAVANI, G. Repertorio metrico della lirica galego-portoghese, Roma, Edizioni dell'Ateneo, 1967). Fue precisamente el uso de esta base de datos lo que hizo recaer nuestra atención en los aspectos que aquí comentamos.

${ }^{3}$ De estas particularidades nos ocupamos en otro trabajo (en prensa): "Esquemas rimáticos y cantigas de refrán". 


$\begin{array}{ccc}\text { I } & \text { II } & \text { III } \\ \text { Coraçon } & \text { desemparar } & \text { estar } \\ \text { forçar } & \text { enton } & \text { son } \\ \text { prison } & \text { non } & \text { enton } \\ \text { tornar } & \text { cobrar } & \text { levar } \\ \text { amor } & \text { meus } & \text { mayor } \\ \text { senhor } & \text { seus } & \text { pavor } \\ \text { matar } & \text { cantar } & \text { mostrar }\end{array}$

Todo ello desemboca en un esquema común a II y III (abbacca), con otro claramente diferente para I (ababccb), ambos con una presencia relativamente frecuente en el corpus gallegoportugués. De este modo, II y III podrían ser consideradas cobras doblas (con variación en c), y la I resultaría consecuente formalmente con el cambio que anuncia también desde un punto de vista significativo, puesto que es en esa estrofa donde el trovador declara que ha encontrado "nov'amor" y "nova senhor" (sintagmas destacados también por su función de rimantes).

2. La cantiga de amor de Pero Garcia d'Ambroa Grave dia naceu, senhor $(126,4)$, transmitida únicamente por B73, presenta múltiples problemas, además de su inusual longitud. ${ }^{4}$ Entre ellos cabe destacar lo que parece un error de copia en las dos últimas estrofas, puesto que los dos últimos versos de la cuarta aparecen copiados como si fueran los dos primeros de la quinta, pero en el siguiente se advierte que está sin dibujar la capital inicial de estrofa. Tal y como ha llegado hasta nosotros,

\footnotetext{
${ }^{4}$ Sólo hay otras dos cantigas de amor de cinco estrofas: Senhor, que coitad' og' eu no mundo vivo, de Nun'Eanes Cêrzeo $(104,9)$ y la dialogada Mia senhor, vin-vus rogar, de Airas Monis d'Asme (13,1), posible escarnio de amor. El número total de composiciones con cinco estrofas en el corpus gallego-portugués es de nueve.
} 
todos los versos son octosílabos de rima masculina, pero la distribución de las rimas difiere en cada una de las estrofas; de hecho, Tavani identifica en su $R M$ cinco esquemas diferentes, dos de ellos -los dos últimos- únicos:

I

ababcca ababbba

senhor senhor

partir falar

devedor sabor

servir matar

partirei

alongarei preguntar

sabor pavor
III abbaccb dizer ali temi

morrer morrerei

malbaratei
IV

abbaaac ababacb averei servir oer tever averei cerrarei filharei serei guisarei i desamor sei partir matar

Las cuatro primeras estrofas podrían ser consideradas, en cierto modo, doblas (I = II, $\left.{ }^{5} \mathrm{III}=\mathrm{IV}\right)$ con una pequeña variación, ${ }^{6}$ pues la segunda prescinde de una rima $c$ para repetir en su lugar la $b$, y la cuarta repite en esos versos 5 y 6 la rima $a$, reservando la $c$ para el último verso, a modo de palabra perduda (aunque coincide estrictamente con la rima $a$ del último verso de I y II). La quinta estrofa quedaría sin pareja; en los cuatro primeros versos, reproduce el esquema de I y II, presenta una aparente palabra

${ }^{5}$ Unidas también por la palabra-rima del v. 1, senhor.

${ }^{6}$ En sentido estricto, en I - II sólo es realmente "doble" la rima $a$, pues $b$ (-ir / -ar) varía, y I añade una rima $c$ (-ei) que luego será reutilizada en las estrofas IV y V. En III - IV sólo sería "doble" (parcialmente) el esquema, pues, aunque coincide la rima -ei, aparece empleada en versos diferentes (es $c$ en III y $a$ en IV); la rima - er $=a$ (III) debería contener un /e / cerrado, opuesto al que debería ser abierto en $b$ de IV. La rima -i aparece sólo en la estrofa central y el -or de IV enlaza con $a$ de I y II. 
perduda en el penúltimo verso y utiliza no sólo rimas que aparecen en las estrofas precedentes, sino incluso cuatro rimantes ya empleados previamente. ${ }^{7}$ Las rimas se combinan entre sí a lo largo de la composición del modo siguiente:

$$
\begin{aligned}
& a \mathrm{I}, \mathrm{II}=c \mathrm{IV} \\
& b \mathrm{I}=a \mathrm{~V} \\
& c \mathrm{I}, \mathrm{III}=a \mathrm{IV}=b \mathrm{~V} \\
& b \mathrm{II}=c \mathrm{~V}
\end{aligned}
$$

3. La disposición estrófica de la composición de Vasco Fernandez Praga de Sandin De cuita grande e de pesar $(151,4)$ llamó la atención de Colocci, que anotó en el espacio que la precede en $B 99$ "la prima et l'ultima et le due di mezzo" (fol. 26r), como si se tratase de una variante más "redonda" de las cobras doblas: en lugar de I = II y III = IV (que sólo sería posible alterando el orden que presentan las estrofas en $A$ y $B), \mathrm{I}=\mathrm{IV}$ y II = III. En cualquier caso, no varían sólo las rimas (I = IV: $a=$-ar, $b=$-on, $c=$-er; $\mathrm{II}=$ III: $a=-$ en, $b=-$ er, ${ }^{8} c=-\mathrm{i}$ ), sino también los propios esquemas: I y IV: ababcca / II y III: abbacca; como se puede apreciar, se modifica la primera parte de las estrofas (vv. 1-4), porque la segunda (vv. 5-7) muestra una serie (cca) común a todas.

4. Johan Soarez Somesso entrelaza las rimas de Quero-vus eu ora rogar $(78,21)$ del siguiente modo: ${ }^{9}$

${ }^{7}$ En total, son cinco los rimantes que se repiten (además del senhor ya comentado): partir, servir, sabor, matar y averei.

${ }^{8}$ Frente al que ofrece la rima $c$ de I y IV, este -er tendría que presentar la vocal abierta.

${ }^{9}$ Las únicas rimas que no se repiten son $b$ (-or) de la primera estrofa y a (-i) de la última. 


$$
\begin{aligned}
& a \mathrm{I}=c \mathrm{II}=b \mathrm{III} \\
& c \mathrm{I}=b \mathrm{II}=a \mathrm{III}=c \mathrm{IV} \\
& a \mathrm{II}=c \mathrm{III}=b \mathrm{IV}
\end{aligned}
$$

Además, liga la primera estrofa con la segunda retomando para el verso 5 de ésta el rimante (pesar) del v. 4 de la anterior, y la segunda con la tercera mediante una operación similar: amar, del v. 6 de II reaparece en el v. 2 de III. Entre III y IV, la única relación que logramos establecer es la existente entre desejar (v. 7 de III) y desejei (v. 2 de IV).

El resultado de todas estas combinaciones es un esquema abbaccb, en el que varía tan sólo la rima del último verso de I, que reproduce la del primero en lugar de la del segundo (abbacca), procedimiento similar al que utiliza el mismo trovador en Ben-no faria, se nembrar $(78,3)$, donde se registran los mismos esquemas, ${ }^{10}$ aunque, en este caso, la cuarta estrofa reproduce el esquema de la primera (I y IV = abbacca (II y III = abbaccb), con lo que recuerda la distribución vista en 151,4.

Lo mismo acontece en la cantiga de Nuno Rodriguez de Candarei En gran coita vivo, senhor $(109,2)$, que aparece en $A 68$ y B18bis con sólo tres estrofas (I = abbaccb / II y III: ${ }^{11}$ abbacca); lo curioso es que la misma composición se repite en $B 1451$ y V1061 (en este caso, atribuida a Johan de Gaia -66,2-) con una estrofa adicional, intercalada entre I y III, que reproduce esquema y rimas de I, con lo que configura una cantiga completa en cobras doblas, sin más incidencias reseñables que el

\footnotetext{
${ }^{10}$ Las únicas correlaciones que podemos establecer en este caso son: $a \mathrm{I}=c \mathrm{II}=b$ III $/ b \mathrm{I}=c \mathrm{III}$.

${ }^{11}$ Doblas no sólo por el esquema sino también por la identidad de rimas.
} 
hecho de que la variación de rimas se ve reforzada por esa variación en la rima del último verso en III y IV. ${ }^{12}$

5. Aunque en el espacio disponible entre $A 99$ y $A 100$ cabría una estrofa más, no hay indicios suficientes para considerar que Mais de mil vezes coid'eu no dia, de Pero Garcia Burgalês $(125,18)$, no esté completa con sus dos estrofas singulares en las que no sólo cambian las rimas (manteniéndose en ambas a como "femenina"), sino también la parte final del esquema (ababccbb / ababccdd), por motivos semejantes a los comentados en las cantigas de refrán: los dos últimos versos pueden repetir una de las rimas anteriores o introducir una nueva.

6. Roi Queimado, por el contrario, mantiene inalterados los dos últimos versos de cada una de las tres estrofas singulares de que consta Pois mia senhor me manda $(148,16)$, para variar, en alternancia, la distribución de los cuatro primeros: abbacc, en I y III / ababcc en II. Elige siempre como $a$ lo que podríamos considerar una rima cara "femenina" (-anda / -ende / -endo), establece casi un dobre en I ("me manda", v. 1 - "demanda", v. 4) y entrelaza II y III invirtiendo "vejo" (v. 2) y "entende" (v. 3) de II con sus rimas derivadas "entendo" (v. 1) y "veja" (v. 2) de III.

7. Martin Moxa construye también en cobras singulares la cantiga Que grave coyta que meé dizer $(94,17)$, en la que, recurriendo a una de las opciones empleadas en las composiciones con refrán, modifica la rima del verso final, haciendo que la primera estrofa responda al tipo abbaccb y la segunda a abbacca.

${ }^{12}$ Estructuras similares con cobras singulares se advierten en otras cantigas, como Que ben que m'eu sei encobrir de Nuno Fernandez Torneol $(106,17)$, que prefiere alternar los esquemas con variación: ababcca en I y III, ababccb en II y IV. 
Se trata, en cualquier caso, de un texto bastante elaborado -donde puede apreciarse el motivo del "cambio" (no explícitamente de dama, sino de tierra, en busca de una donde entiendan su "coita")- en el que destacan las palabras "cantar" y "dizer", con su sinónimo "falar"; la primera de ellas aparece como rimante en los vv. 2 y 7 de I y se repite en el v. 5 de II (con lo que II retoma como $c$ la rima $b$ de I), "dizer" finaliza los vv. 1 y 4 de I, y las dos podrían ponerse en relación con el "falar" del v. 6 de II. A su vez, en II, el primer y último verso finalizan con el verbo copulativo "é", mientras que en I las mismas posiciones están ocupadas, respectivamente, por "dizer" y "cantar".

8. Si la versión transmitida por $B 973$ y V560 es correcta, ${ }^{13}$ Airas Engeitado emplea dos esquemas únicos (aabccbdad / aabccbdde) en Tan grave dia vos eu vi (12,4), estructurada en cobras unisonantes (aunque con rima $d$ "singular"). ${ }^{14}$

También Johan de Gaia entrelaza dos esquemas únicos, en este caso de cobras singulares, en Meus amigos, pois me Deus foy mostrar (66,4): abcbdca (I y III) / ababcda (II), los dos con la particularidad de presentar una palabra perduda (la rima $d$ ), a la que en II se suma también $c$.

Estevan da Guarda mantiene un esquema único ababbcca en las tres primeras estrofas de Ora, senhor, tenho muyt'aguysado

${ }^{13}$ Sería posible reconstruir parcialmente en II el esquema de I alterando la lectura de dos de los últimos versos en "nen ouve nunca ben, senhor / nen sei que x'est, avenm'assi" (lo que provocaría palabras rima en los vv. 7 y 8), pero no encontramos una alternativa similar para el v. 9.

${ }^{14}$ Se completa con una tornada de cinco versos (aabcc) que retoma la rima $a$ de I y II (repitiendo en el v. 1 el rimante "assy" presente en el v. 8 de I) y como rima $b$ la que en II aparece como e ("mal" "val"); $c$ es "singular". 
$(30,23)$, construida en cobras singulares, pero lo modifica en la cuarta y última para convertirlo en ababbccb, otro esquema poco frecuente. ${ }^{15}$

9. Un posible problema de transmisión se advierte en Assi me trax coitado, de Don Denis $(25,16)$, que, en la edición de Lang, presenta una estructura en cobras unisonantes con una variación en la distribución de las rimas, pues la estrofa I responde al esquema ababbbb, pero las otras dos ofrecen ababccb. Tanto B531 como V134 copian la segunda estrofa de tal modo que sería posible editar:

Ca viv'en tal cuidado come quen sofredor é de mal aficado que non pode maior, se mi non val a que en forte ponto vi, ca ja de mor$t^{\prime}$ ei prazer e nen un pavor

De este modo, se mantendría el esquema inicial, que podría recuperarse también (aunque aquí no lo presenten los manuscritos) para III:

E faço mui guisado, pois sõo servidor da que mi non dá grado,

${ }^{15}$ Además de Estevan da Guarda, lo emplea Pero Gomez Barroso en Pero d'Ambroa, se Deus me pardon $(127,7)$ y aparece también en V668 (Donna e senhora de grande vallia), copiada al final de las composiciones de Juiäo Bolseiro, pero que se corresponde con una interpolación tardía (V. G. Tavani, "Sobre a atribuição a D. Dinis e a Juião Bolseiro de duas canções tardias", en Ensaios portugueses. Filologia e Lingüística, Imprensa Nacional-Casa da Moeda, Lisboa, 1988, pp. 317-349). 
querendo-lh'eu melhor

ca min nen al; por én, conor-

$t^{\prime}$ eu non ei ja se non da mor-

$t^{\prime}[e]$ ende sõo desejador.

El alambicamiento de la cantiga, con un esquema rimático único y esos encabalgamientos forzados en los versos 5 y 6 , se enriquecería además con la presencia de lo que en la primera estrofa podría ser considerado un dobre ("amor", vv. 2-6), pero que se convierte así en el v. 6 en una palabra-rima ("d'amor" / "da mor-") que juega con una paronomasia bien conocida.

10. En dos posibles escarnios de amor, Johan Garcia de Guilhade utiliza las variaciones de forma distinta. En Cuydous'amor que logo me faria $(70,13)$, que sigue el modelo de las cobras unisonantes, mantiene la distribución abbacca en las dos primeras estrofas, pero la altera en la tercera para repetir $b$ en los vv. 5 y 6 (abbabba). ${ }^{16}$ En cambio, en Deus! Como se foron perder e matar $(70,14)$, compuesta en cobras singulares, modifica la rima de los dos últimos versos en todas las estrofas: mantiene los cuatro primeros versos como abab, repite $a$ en la primera estrofa (ababaa), $b$ en la segunda (ababbb) e introduce una tercera rima en la última (ababcc). ${ }^{17}$

${ }^{16} \mathrm{La}$ "irregularidad" se corregiría fácilmente si se pudieran intercambiar los tres últimos versos de III con la tornada, que repetiría así las rimas $a$ y $b$ de las estrofas (bba), mientras que, tal y como ha sido transmitida por $A 238$, la fiinda presenta en los dos primeros versos una rima nueva y sólo mantiene los lazos con las estrofas a través del último verso, que coincide con la rima $a$.

${ }^{17}$ En cualquier caso, en III repite "alguen" como rimante para $c$, "veer" en el v. 6 de II y el v. 4 de III, y el último verso de III es una variación paralelística del último de II. 
11. Entre las cantigas de amigo, el único caso reseñable que hemos encontrado en la modalidad "de mestría" es Sey eu, donas, que non quer tan gram bem, de Pedr'Amigo de Sevilha $(116,34)$, que combina las cobras singulares con una especie de "cobras alternadas" en lo relativo no sólo a la distribución de los esquemas sino también a la de las propias rimas entre II y IV: abbcac (I y III) / aaabab (II y IV); la rima $a$ (-er) de II funciona como $b$ de IV, y la rima $b$ (-ar) de II como $a$ de IV.

12. Son más abundantes los ejemplos en el bloque de las cantigas de escarnio y maldecir. Así, Pero non fui a Ultramar, de Martin Soarez $(97,28)^{18}$ emplea cobras singulares pero entrelaza las rimas del siguiente modo:

$$
\begin{aligned}
& a \mathrm{I}=b \mathrm{II} \\
& b \mathrm{I}=a \mathrm{II} \\
& a \mathrm{III}=b \mathrm{~V} \\
& b \mathrm{III}=a \mathrm{~V} .
\end{aligned}
$$

Como puede apreciarse, la única estrofa que ofrece rimas diferentes es IV, que es precisamente la que presenta una variación (abbaccb) en el esquema abbabba común a todas las demás. Obien su singularidad obedece a una intención declarada de llamar la atención sobre esa estrofa o bien fue interlacada en un momento posterior, porque las particularidades no se limitan a lo ya comentado, sino que es asimismo la única estrofa que no mantiene otras relaciones con las inmediatas (es más, "interrumpe" la secuencia -a modo de doblas- que liga la III a la V): el v. 2 de I ("muito sei eu a terra ben") tiene su correlato en el v. 1 de II ("E as

18 Transmitida únicamente por B143, ofrece, entre otras cosas, la particularidad de que todas las estrofas fueron copiadas "en prosa", y es el propio Colocci el que parece haber ido señalando con un ángulo el corte de los versos. 
jornadas seieu ben"): el v. 2 de III ("que vio prender Nostro Senhor") en el v. 1 de V ("Por i andou Nostro Senhor") y el v. 3 de III ("e averedes i gran sabor") en el v. 4 de V ("o perdon, ouve gran sabor").

13. Ao daian de Cález eu achei, de Alfonso X $(18,4)$ repite un procedimiento ya visto en varias cantigas: modifica la distribución ababccb de las cuatro primeras estrofas (construidas como cobras doblas, $\mathrm{I}=\mathrm{II}, \mathrm{III}=\mathrm{IV}^{19}$ ) en el último verso de la quinta y última (ababcca), que retoma no sólo la rima del primero, sino también el rimante ("al").

14. Airas Nunez inicia O meu señor, o bispo, na Redondela ũu dia $(14,10)$ con un esquema poco usual aaabbb,$^{20}$ que modifica en las otras $\operatorname{dos}^{21}$ con la introducción de una palabra perduda en el penúltimo verso de II y III, que adoptan así una distribución aaabcb única en el corpus gallego-portugués y en la que las rimas $a y c$ son singulares, mientras que $b$ se comporta como unisonante.

15. Martin Moxa, a mia alma se perça, de $\mathrm{A}^{\circ}$ Gomez, "jograr de Sarria" $(5,1)$, presenta dificultades de copia en lo relativo al corte de los versos, pero, al menos en la edición de Lapa seguida en $M e d D B$, alterna la distribución abbacca de I y III con la alternativa ababccb de II. El engarce de II con I se logra mediante la repetición de la rima $c$ (-er), en una estructura de

${ }^{19}$ El rimante "faz" del v. 4 de III reaparece en el v. 2 de IV, y el "ten" se reitera en el v. 6 de III y el v. 5 de IV.

${ }^{20}$ Fuera de esta cantiga, sólo se encuentra en la única estrofa conservada de la cantiga de amigo Nunca eu vi melhor ermida, nen mais santa de Martin de Ginzo $(93,6)$.

${ }^{21}$ En realidad, en $B 885$ falta el penúltimo verso de II, y tanto este testimonio como V468 presentan algunas dificultades de lectura en los rimantes de la última estrofa. 
cobras singulares en la que se logra que el último verso de cada estrofa rime con el v. 4 respectivo. ${ }^{22}$

Es también la estrofa central la que emplea Johan Soarez Coelho para introducir una variación en Joan Fernandiz, mentr'eu vosc'ouver $(79,27)$, pues I y III responden al esquema abbcca (y la rima $c$ de III reitera la $b$ de I, -ei), mientras que II se construye como abaccb.

En cambio, este mismo trovador organiza en cobras doblas (ababaab) las dos primeras estrofas de Maria do Grave, grav'é de saber $(79,34)$, y lo convierte en abbacca en III, aunque manteniendo como rima $a$ de esta última estrofa la que en las dos anteriores era $b$, constituida por un rimante "equívoco" ("grave") que se repite tres veces (vv. 2, 4 y 7 de I y II; vv. 1, 4 y 7 de III), e introduciendo una tercera rima que no había en las anteriores.

A idéntico resultado abbacca llega Coelho en la tercera estrofa $^{23}$ de Joan Garcia tal se foi loar $(79,28)$, que había iniciado en cobras doblas (c es singular) como abbaccb en I y II; las dos primeras estrofas están entrelazadas, además, por el rimante "queixar" (vv. 4 de I y 1 de II), y la segunda y tercera por "trobador" (v. 5 de II y III), que se repite en plural ("trobadores") en el v. 2 de III; la presencia de esta especie de "palabra-rima" provoca que $c$ (elemento diferenciador entre I y II) resulte ahora unisonante entre II y III.

16. Pero Garcia d'Ambroa emplea un esquema ababccb en las estrofas I, II y IV de De Pero Bõo and'ora espantado $(126,3)$, pero

${ }^{22}$ La primera estrofa ofrece la particularidad de una rima $a$ asonante (perça - erva -Eva), cuando todas las demás son consonantes.

${ }^{23}$ En este caso sigue una tornada de tres versos, que reproduce en vv. 1 y 2 la rima $c$ de I, y en v. 3 no sólo la rima $a$ de III, sino también su último rimante ("dereito"). 
lo altera en III convirtiéndolo en abbacca. ${ }^{24}$ La ligazón entre las cuatro cobras singulares se logra gracias a la repetición "alternada" de una de las rimas, de tal modo que $a$ I (-ado) se retoma como $a$ III, y $b$ II (-ia) como $c$ IV.

La introducción de un cambio en la última estrofa (ya visto en otras ocasiones) es el recurso utilizado por Afons'Eanes do Coton en Paai Rengel e outros dous romeus $(2,17)$, donde altera la ordenación ababccb de I y II en abbacca de III. Los esquemas coinciden con los que ofrece la cantiga 5,1 comentada más arriba y, como allí, el cambio de rima en el último verso parece responder al intento consciente de vincularlo al cuarto, el cual -como resultado de la alternancia entre las secuencias iniciales básicas abab y abba- unas veces coincide con el primero y otras con el segundo.

Cumple el mismo requisito (y repite el segundo de esos dos esquemas) otra composición del mismo trovador, Mestre Nicolás, a meu cuidar $(2,15)$, donde es también la tercera estrofa la que presenta una variación (abbaaaa, única); pero aquí la pieza se completa con una cuarta estrofa, con el mismo esquema que I y II y estas, a su vez, retoman de forma alternada las rimas -ar $(a \mathrm{I}, b \mathrm{II})$ y -er $(b \mathrm{I}, a \mathrm{II})$.

17. Ena primeira rua que cheguemos, de Martin Anes Marinho $(89,1)$ presenta varios elementos de difícil explicación. Por una parte, la segunda estrofa abre y cierra con el rimante "capa" (uno de los motivos centrales de la burla), pero ese dobre no se produce en ninguna de las cinco estrofas restantes; por otra, VI parece una

\footnotetext{
${ }^{24}$ Adviértase cómo, en estos últimos casos, una de las constantes parece ser la identidad rimática del v. 7 (y último) con el v. 4 (el que ocupa la posición central de la estrofa y marca la transición entre lo que normalmente pueden considerarse las dos subdivisiones del esquema).
} 
reelaboración de IV, de la que reproduce-en las mismas posicionestodos los rimantes $a$ y $b$ (y es posible que, al menos en un caso, también $c$ ); y la rima $b$ de IV y VI (-ada) es usada como $a$ en V (que, a su vez, se inicia como "capdenal" de II, con un primer hemistiquio presente también en el v. 5 de III). En cuanto a los esquemas, el "común" es, en este caso, ababcca, que se convierte en ababccb (una vez más, el v. 7 recupera la rima del v. 4) sólo en III.

18. Pero da Ponte dispone en cobras doblas (ababccb) las dos primeras estrofas de Aos mouros que aqui son $(120,4)$, pero en la tercera y última repite la rima $a$ en los vv. 5 y 6 , provocando la variación rimática ababaab. La relación entre I y II se consolida con la repetición del rimante "ouver" en los vv. 5 de I y 6 de II, y con la posible equivocatio entre "raçon" (v. 3 de I) y "razon" (v. 3 de II); pero también se establecen lazos entre I y III, pues el rimante "dan" del v. 7 de III cambia sólo el número de la tercera persona del v. 2 de I ("da") y el tiempo verbal del v. 4 de I ("darlhis-á"), del mismo modo que el infinitivo "filhar" del v. 3 de III "recuerda" el futuro "filhará" del v. 7 de I.

De modo inverso opera en Noutro dia, en Carrion $(120,29)$, donde es la estrofa inicial ababaab la que se transforma en ababccb en las tres restantes; aunque la pieza está compuesta en rimas singulares, sólo I se mantiene claramente diferenciada, pues entre las demás se produce la siguiente combinación:

$$
\begin{aligned}
a \mathrm{II}= & c \text { III, IV ("aquí" del v. } 3 \text { de II se retoma en el v. } 6 \\
& \text { de IV) } \\
b \mathrm{II}= & a \text { III (los rimantes de III pertenecen al mismo } \\
& \text { verbo: "achei", v. } 1-\text { "acharei", v. } 3) \\
b \mathrm{III}= & a \text { IV. }
\end{aligned}
$$

En Eu, en Toledo, sempr'ouço dizer $(120,17)$, el mismo Pero da Ponte combina la secuencia abbacca (I y II) con la variante abbaccb (III), refuerza la ligazón entre I y II repitiendo el rimante "dizer" en 
los vv. 1 de I y 4 de II, pero facilita también el paso de II a III repitiendo "prender" del v. 7 de II como "aprender" en el v. 1 de III; y mantiene unisonante en las tres estrofas la rima $a$ (-er).

También en uno de los escasos ejemplares conservados de lo que se considera "cantiga encomiástica" $(120,30)$, este trovador emplea el esquema ababccb en las tres primeras estrofas y-como en tantos otros casos-introduce una variación en la última (seguida de dos fiindas): ababcca. ${ }^{25}$

19. Afonso Lopez de Baian elabora de forma compleja su escarnio En Arouca ũa casa faria $(6,3)$, que abre y cierra con el mismo rimante ("faria", vv. 1 de I y 7 de III), dispone como unisonantes las rimas $a$ y $b$ y como singular $c$, y altera la distribución ababcca de I en abbacca en II y III. La estrofa central reitera el rimante "dia" en los vv. 4 y 7 (una vez más, la correlación de rimas entre esos dos versos, aquí reforzada por el propio rimante), pero las tres están también entrelazadas por la repetición inversa de "aver" (vv. 4 de I, 3 de II y 2 de III), y el "fazer" (ligado, a su vez, morfológicamente, a "faria") del v. 2 de I reaparece como v. 3 de III:

${ }^{25}$ Las estrofas son singulares, pero la rima $c$ de II (-ei) y III (-eis) están tan emparentadas que el v. 6 de ambas estrofas presenta la forma singular y plural del mismo sustantivo: "rey" (III) / "reys" (IV). La segunda fiinda repite en el primer verso el rimante "conquereu" del v. 5 de IV. La primera estrofa, por su parte, juega con la conocida paronomasia "amar" (v. 4) - "a mar" (v. 7). 


$\begin{array}{ccc}\text { I } & \text { II } & \text { III } \\ \text { faria } & \text { serviria } & \text { Maria } \\ \text { fazer } & \text { prazer } & \text { aver } \\ \text { recearia } & \text { aver } & \text { fazer } \\ \text { aver } & \text { dia } & \text { descobrila-ia } \\ \text { cal } & \text { madeirar } & \text { mester } \\ \text { al } & \text { telhar } & \text { der } \\ \text { queria } & \text { dia } & \text { faria }\end{array}$

20. Pedr'Amigo de Sevilha organiza las dos primeras estrofas de Hun cavaleyro, fi' de clerigon $(116,37)$ a modo de cobras doblas, pero alterando ligeramente el esquema, pues el tercer verso de la segunda (que repite el rimante "non" del v. 5 de I) reproduce la rima del primer verso en lugar del segundo, dando lugar a la variación abbcac (I) / abacac (II); la tercera estrofa (que queda libre, como es usual en estos casos) retoma como $b$ la rima $c$ (-er) de I y II, pero vuelve a modificar el esquema: abacbc.

21. Martin Moxa compone una cantiga moral $(94,2)$ empleando una estructura no repetida en ningún otro texto del corpus (aabaabcccbcccb), y prescinde en la cuarta estrofa ${ }^{26}$ de la tercera rima diferente para reiterar en su lugar $a$ : aabaabaaabaaab. La rima $b$, a su vez, establece una relación de cobras dobras, pues en I y II es -ar, y en III y IV -er; además, la transición entre los dos pares se ve facilitada por la proximidad de la rima $c$ en II (-adas) y III (-ados), que permite incluso la alternancia morfológica entre "achegadas" (v. 13 de II) y "achegados" (v.11 de III). Las estrofas III (v. 3) y IV (v. 6) repiten también el rimante "poder".

${ }^{26}$ La fiinda, de cuatro versos con rimas aaab, repite las rimas de esta última estrofa $(a=-\mathrm{ia}, b=-\mathrm{er})$ 
22. También las tensos recurren en ocasiones a variaciones similares a las que hemos visto hasta ahora.

Así, la primera intervención (es decir, el primer par de cobras doblas) de los "contendientes" Johan Perez d'Aboim y Johan Soarez Coelho en Joan Soarez, comecei $(75,8=79,29)$ utiliza el conocido esquema ababccb, pero la segunda pareja de estrofas lo convierte en su variante ababcca.

La disputa entre Estevan da Guarda y Don Josep (30,35 = $84,1)$ parece tener un problema de ordenación: o bien falta (como supone Lapa) una estrofa entre la cuarta y la quinta (y, en este caso, faltaría también una octava, para mantener el equilibrio entre ambas intervenciones), o bien las estrofas copiadas en $B 1315$ y V920 como quinta y sexta están en realidad invertidas, ${ }^{27}$ porque el orden de interlocución que presentan en los manuscritos es: EG - DJ - EG - DJ - DJ - EG. La segunda opción es perfectamente viable si prestamos atención a las rimas, pues la estructura de cobras doblas muestra claramente la relación entre $\mathrm{V}$ y VI, una relación que se ve incrementada por la reiteración de los rimantes "fiz" (vv. 4 de V y 1 de VI) y "negado" (vv. 5 de V y 2 de VI). En I y II, las rimas son: $a=-a r$, $b=-$ ores, $c=-$ eu; en III - IV,$a=-$ on, $b=$-ade (III) $/$-ado (IV), $c=$ -or; en V - VI, $a=$-iz, $b=$-ada (V) / -ado (VI), $c=$-ado. Esta secuencia indica ya una pequeña variación en la segunda pareja que, al reproducirse en la tercera, provoca que el esquema abbacca se convierta en la sexta estrofa en abbabba; de todos modos, no se puede descartar la existencia de problemas de transmisión enmendables que permitan recuperar una rima -ado para $b$ en III y IV, y -ada en la misma posición para V y VI, con lo que el resultado sería totalmente "regular".

${ }^{27}$ Colocci debió de advertir la existencia de algún tipo de problema porque, en el fol. $281 \mathrm{v}$ de $B$, a la altura del primer verso de V, dibujó en el margen izquierdo su típico signo de advertencia (+). 
Si resumimos cuanto hemos comentado en una tabla y prescindimos del resto de artificios que los trovadores combinan con la variación rimática (contribuyendo con ello a enriquecer la cantiga desde el punto de vista formal y, en ocasiones, a reforzar su propio contenido), la situación con la que nos encontramos es la siguiente:

\begin{tabular}{|c|c|c|c|c|c|c|}
\hline trovador & ctga & esqI & esqII & esqIII & esqIV & $\operatorname{esq} V$ \\
\hline Osoir'Anes & 111,1 & ababccb & abbacca & & & \\
\hline $\begin{array}{l}\text { Pero Garcia } \\
\text { d'Ambroa }\end{array}$ & 126,4 & ababcca & ababbba & $\mathbf{a b b a c c b}$ & $\mathbf{a b b a a a c}$ & ababacb \\
\hline $\begin{array}{l}\text { Vasco F. Praga } \\
\text { de Sandin }\end{array}$ & 151,4 & ababcca & abbacca & & & \\
\hline $\begin{array}{l}\text { Johan Soarez } \\
\text { Somesso }\end{array}$ & 78,21 & abbaccb & abbacca & & & \\
\hline $\begin{array}{l}\text { Johan Soarez } \\
\text { Somesso }\end{array}$ & 78,3 & abbacca & abbaccb & & & \\
\hline $\begin{array}{l}\text { Nuno R. de } \\
\text { Candarei }\end{array}$ & 109,2 & abbaccb & abbacca & & & \\
\hline $\begin{array}{l}\text { Nuno Fernandez } \\
\text { Torneol }\end{array}$ & 106,17 & ababcca & ababccb & & & \\
\hline $\begin{array}{l}\text { Pero Garcia } \\
\text { Burgalês }\end{array}$ & 125,18 & ababccbb & ababccdd & & & \\
\hline Roi Queimado & 148,16 & abbacc & $\mathbf{a b a b c c}$ & & & \\
\hline Martin Moxa & 94,17 & abbaccb & abbacca & & & \\
\hline Airas Engeitado & 12,4 & aabccbdad & aabccbdde & & & \\
\hline Johan de Gaia & 66,4 & abcbdca & ababcda & & & \\
\hline Estevan da Guarda & 30,23 & ababbcca & ababbccb & & & \\
\hline Don Denis & 25,16 & ababbbb & ababccb & & & \\
\hline $\begin{array}{l}\text { Johan G. de } \\
\text { Guilhade }\end{array}$ & 70,13 & ababaa & ababbb & ababcc & & \\
\hline $\begin{array}{l}\text { Pedr'Amigo de } \\
\text { Sevilha }\end{array}$ & 116,34 & abbcac & $\mathbf{a} \mathbf{a} \mathbf{a b} \mathbf{a b}$ & & & \\
\hline Martin Soarez & 97,28 & abbabba & abbaccb & & & \\
\hline Alfonso X & 18,4 & ababccb & ababcca & & & \\
\hline Airas Nunez & 14,10 & aaabbb & aaabcb & & & \\
\hline $\mathrm{A}^{\circ}$ Gomez & 5,1 & abbacca & ababccb & & & \\
\hline $\begin{array}{l}\text { Johan Soarez } \\
\text { Coelho }\end{array}$ & 79,27 & abbcca & abaccb & & & \\
\hline
\end{tabular}




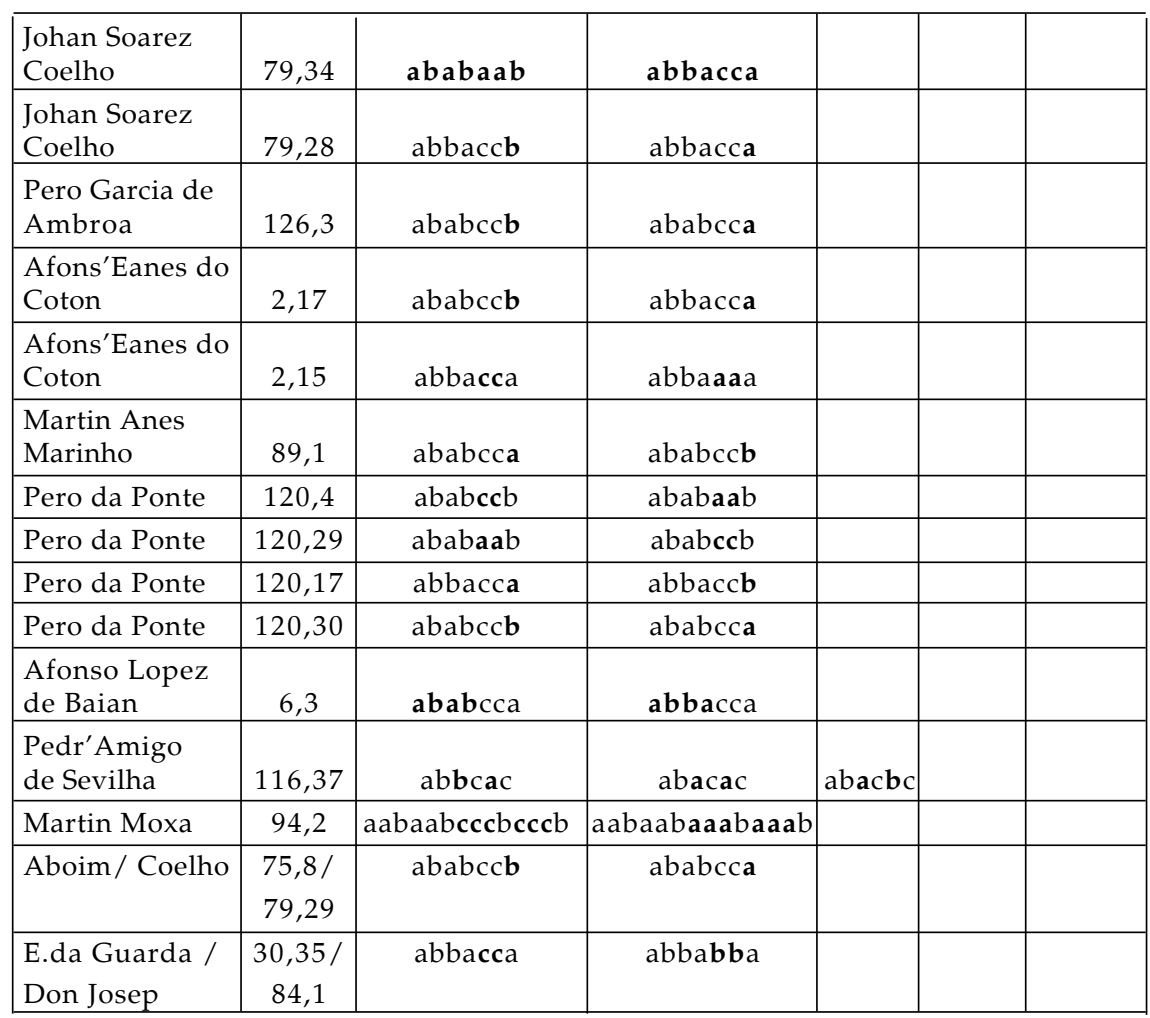

Algunos casos, como los de las cantigas 126,4 o 94,2, son realmente singulares y merecedores de un estudio particularizado, pero la mayoría de los restantes parecen responder a criterios distributivos como los siguientes:

(1) En las estrofas de siete versos, ${ }^{28}$ puede establecerse un corte estructural entre los cuatro primeros y los tres restantes. ${ }^{29}$

${ }^{28}$ En estrofas de más de siete versos -y dejando a un lado la de Martin Moxa-, puede advertirse un mínimo cambio en la rima del último verso, como 30,23, o que la modificación se extienda a los dos últimos $(12,4,125,18)$.

${ }^{29}$ Recordemos que el cuerpo de la estrofa que precede al estribillo en la mayoría de las cantigas de refrán suele contener precisamente 
Para el primer bloque, las combinaciones rimáticas habituales son abab y abba, que podrían considerarse intercambiables, como acontece en las cantigas 111,1, 151,4, 5,1,79,34 o 6,3.

En cuanto al segundo, pueden producirse al menos las siguientes modificaciones:

(a) A una secuencia cc puede seguir un verso final combinable bien con la primera rima de la estrofa bien con la segunda, dando lugar a esquemas del tipo ababcca / ababccb o abbacca / abbaccb; las cantigas 78,21, 78,3, 109,2, 106,17, $94,17,18,4,79,28,126,3,2,17,89,1,120,17,120,30$ y la tensón entre Aboim y Coelho se limitan a establecer una variación en el último verso, que en unas estrofas presenta la rima $a$ y en otras la $b$. Otras composiciones combinan esta alteración con la anterior, como puede verse en 111,1 o 5, $1^{31}$ (en ambos casos se produce una alternancia entre $\mathbf{a b a b c c b}$ y abbacca), que aseguran la rima del último verso de la estrofa con el cuarto (último del primer bloque).

(b) En otros casos $(25,16,2,15,120,4,120,29,30,35 / 84,1)$ es esa tercera rima cc la que puede omitirse para volver a emplear una de las presentes en la primera parte de la estrofa (ababccb / ababbbb, abbacca / abbaaaa, ababccb / ababaab, abbacca / abbabba). Aunque resulte menos frecuente, esta modalidad puede complicarse cambiando

cuatro versos. La única excepción reseñable que registramos en este sentido es la cantiga 66,4, que introduce una variación de distinto signo en el primer bloque, acompañada de otra igualmente significativa en el segundo: ababcda / abcbdca,

${ }^{30}$ La artificiosidad de esta cantiga de Johan Soarez Coelho complica mucho más los esquemas, puesto que casi lo único que se mantiene igual es que el último verso rima con el cuarto (ababaab / abbacca). ${ }^{31}$ Y, de modo diferente, en 79,34 (vid. supra nota 29). 
también la rima del último verso, como en 97,28 (abbaccb /abbabba).

(2) En estrofas de seis versos, la bipartición señalada no parece tan clara, pues, si dejamos a un lado los casos de 148,16 (que se comporta como las cantigas 151,4 etc.) y 70,13 (que varía el segundo bloque tres veces), las posibilidades combinatorias se registran en varias posiciones diferentes: abbcac / aaabab (116,34, que parece seguir la tendencia a hacer rimar el último verso con el cuarto), abbcca / abaccb $(79,27)$, abbcac / abacac / abacbc $(116,37)$.

Aunque no todos los trovadores que recurren a este artificio pertenezcan a la misma generación, sí existen relaciones conocidas (participación en los mismos ciclos escarninos, por ejemplo) entre buena parte de ellos, por lo que estas alteraciones del esquema rimático (en ocasiones, vinculadas a otros recursos ${ }^{32}$ ) pueden haber sido una práctica relativamente habitual con la que tal vez pretendían dar muestra de su capacidad ${ }^{33}$ para adaptar un único esquema melódico ${ }^{34}$ a diferentes combinaciones rimáticas con las que producían estructuras únicas en la lírica gallego-portuguesa.

\footnotetext{
32 Vid. BILLY, Dominique. L'arte delle connessioni nei trobadores. In: BILLY, Dominique; CANETTIERI, Paolo; PULSONI, Carlo; ROSSELL, Antoni. La lirica galego-portoghese. Saggi di metrica e musica comparata. Roma: Carocci, 2003. p. 11-111.

${ }^{33}$ Recordemos que algunos de estos trovadores son autores de escarnios -o participan en tensós- en las que se satirizan precisamente las escasas dotes para trovar de.

${ }^{34}$ Las composiciones recogidas en $A$ reservan espacio para la notación musical tan sólo en la primera estrofa, como es habitual cuando esa estructura se repite invariable en todas las demás (con la única excepción posible de la fiinda).
} 
En cualquier caso, lo que no parecen estas muestras son defectos achacables a una falta de maestría de sus autores, sino más bien todo lo contrario, puesto que los resultados, tal vez precisamente por ser nuestros trovadores "poco inclini a sottomettersi a schemi rigorosi", acaban siendo auténticas "esperienze singolari". ${ }^{35}$

\section{Resumo}

A consulta da base de dados MedDB em www.cirp.es permite advertir a existência de um grupo relativamente importante de cantigas que apresentam algum tipo de alteração na suposta norma de correspondência métrica, rimática e melódica entre todas as estrofes da composição (exceto, naturalmente, a fiinda). Esta aportação presta atenção às mudanças deste tipo que se produzem na disposição das rimas no grupo mais difícil de classificar, o das cantigas de mestria, e trata de explicar como se produzem as modificações, ao mesmo tempo em que chama a atenção sobre o fato de que não se tratam de erros cometidos pelos trovadores, mas precisamente do contrário: a introdução de traços inovadores que mostrem a sua capacidade para a composição.

${ }^{35}$ Utilizamos expresiones tomadas de BILLY. L'arte, p. 92. 


\section{Resumen}

La consulta de la base de datos MedDB en www.cirp.es permite advertir la existencia de un grupo relativamente importante de cantigas que presentan algún tipo de alteración en la supuesta norma de correspondencia métrica, rimática y melódica entre todas las estrofas de la composición (excepción hecha, naturalmente, de la fiinda). Esta aportación presta atención a los cambios de este tipo que se producen en la disposición de las rimas en el grupo más difícil de clasificar, el de las cantigas de mestría, y trata de explicar cómo se producen las modificaciones, a la vez que llamar la atención sobre el hecho de que no se trata de faltas cometidas por los trovadores, sino precisamente de lo contrario: de introducir rasgos innovadores que muestren su capacidad para la composición. 